\title{
Image-based cell-size estimation for baculovirus quantification
}

\author{
Tõnis Laasfeld, Sergei Kopanchuk, and Ago Rinken \\ University of Tartu, Institute of Chemistry, Tartu, Estonia
}

BioTechniques 63:161-168 (October 2017) doi 10.2144/000114595

Keywords: baculoviridae quantification; baculovirus expression vector system; Sf9 cells; bright-field microscopy; machine/computer vision

Supplementary material for this article is available at www.BioTechniques.com/article/114595.

Measurement of virus concentration is essential for effective virus-based transfection technologies. Here, we describe a user-friendly, image-based cell-size estimation (ICSE) assay for baculovirus quantification that relies on automated determination of cell diameters from bright-field microscopy images. In the ICSE assay, microplate-based imaging systems and our custom ICSE-Tools software enable measurement of cell morphological parameters over time. Results from the ICSE assay were in agreement with virus concentration measurements obtained using the traditional plaque assay as well as the Coulter principlebased cell-size measurement assay. ICSE-Tools is designed for data organization and image analysis from microplate-based imaging systems, and is freely available at www.gpcr.ut.ee/software.html.

Production of recombinant proteins in mammalian cells has been an essential technology over the last few decades for biomedical studies as well as for the development of biopharmaceutical products. Although numerous cell lines that stably express recombinant proteins of interest have been developed, transient transfection of genes is still an essential technique (1). Transient transfection has a clear advantage if different cell lines need to be studied or if modifications of recombinant proteins have to be produced. Numerous physical and chemical methods have been developed to transfer DNA into cultivated mammalian cells, but achieving stable and reproducible results has remained a challenge (2).

Despite progress in non-viral transfection strategies, virus-based technologies are often preferred. Among these, baculovirus (BV)-based expression systems can induce production of recombinant proteins in both insect cells, for example using the baculodisplay BV expression vector system (BEVS), and mammalian cells, for example using BacMam system (3). Display of recombinant proteins on the surface of BV particles allows for their use as immunogens for developing monoclonal antibodies (4), which is useful for the development of BV-based vaccines (5). G-protein-coupled receptors that are displayed on virus particles are useful for the characterization of receptor complexes $(6,7)$ and ligand binding to these receptors $(8,9)$. The advantages of using the BV system are clear when studying the binding of fluorescent ligands or nucleotides in homogeneous assay systems $(10,11)$.

The ability of modified BVs containing mammalian regulatory sequences (BacMam viruses) to deliver gene vectors to mammalian cells (12) significantly broadens the range of BV applications. Viruses have several advantages over other methods, including their ease of use, large cloning capacity, the possibility of transfecting a cell with multiple viruses, and their low biohazard risk (3). Therefore, BacMam viruses have become a valuable tool for cell assays (13), such as the lucif- erase enzyme fragment complementation assay (14) or the cAMP detection biosensors (15).

The efficiency of recombinant protein production, as well as the overall behavior of transfected cells, is highly dependent on the multiplicity of infection (MOI) (16). Therefore, to obtain reproducible results under optimal conditions, BVs have to be reliably quantified for each assay.

Several assays are available to quantify viruses based on plaque infectivity or cell viability, in addition to antibody-based assays, flow cytometry assays, and quantitative real-time PCR (16). Although these assays are useful, each has its drawbacks (17). A comparison of different methods revealed that the cell-size assay (18) performs well with a reasonable standard error (SE) and is recommended for the determination of virus titer (16). However, there are only a few methods available for obtaining direct data regarding cell size, and even fewer are used for BV quantification. To overcome this problem, we developed an imagebased cell-size estimation (ICSE) assay

The image-based cell-size estimation (ICSE) assay is a microscopy-based method for quantification of baculoviruses (BVs) based on the characteristic increase in size of infected Sf9 cells. Images are analyzed using our custom MATLAB-based software, ICSE-Tools, which detects and measures cell diameters and organizes data based on the experiment description. 


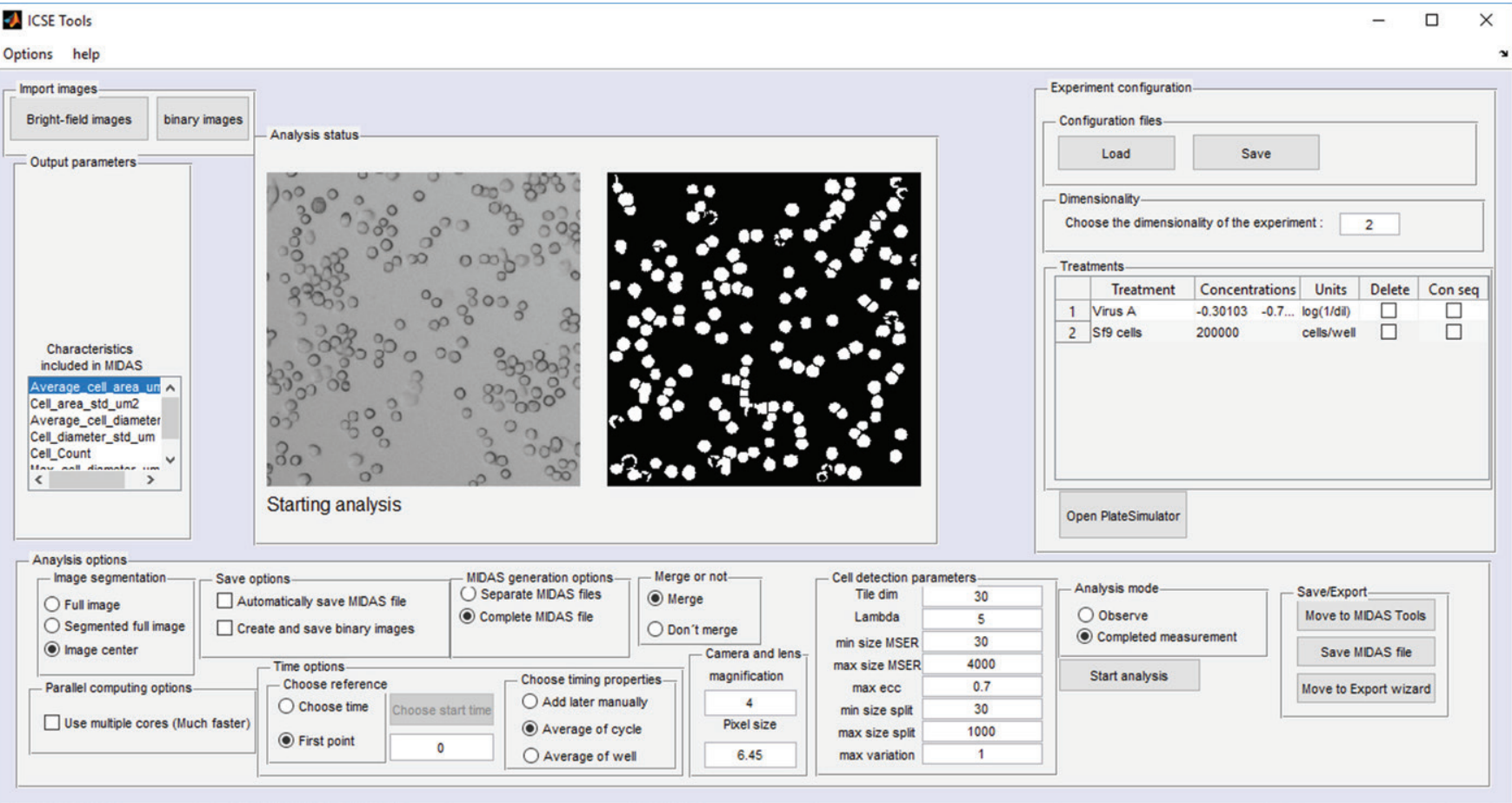

Figure 1. Screenshot of the ICSE-Tools software graphical user interface. Panels are provided for the input of experimental details (Experiment configuration panel), analysis options (Analysis options panel) and data export (Save/Export panel). The software can be downloaded at www.gpcr.ut.ee/software.html, and more information can be found at www.gpcr.ut.ee/ICSETools/ICSEToolsHelp/ICSE.html.

that uses automated microscopes/cell imaging readers allowing for automation of BV quantification. To validate our ICSE assay, we compared it with the Coulter principle-based cell-size estimation assay (CPCSE) and the plaque assay.

\section{Materials and methods}

Baculovirus constructs

The Sf9 cell line from Spodoptera frugiperda pupal ovarian tissue (ThermoFisher Scientific, Waltham, MA) was cultivated for all experiments at $27^{\circ} \mathrm{C}$ in EX-CELL 420 serum-free medium for insect cells (referred hereafter as the medium) (Sigma-Aldrich Chemie GmbH, Darmstadt, Germany), as described previously (19). BVs encoding melanocortin 4 receptor $\left(\mathrm{MC}_{4} \mathrm{R}\right)$ and dopamine 1 receptor $\left(D_{1} R\right)$ were prepared as described previously $(19,20)$, and BacMam BVs containing sequences for different CAMP biosensors, which were developed by Kees Jalink's laboratory (21), were prepared as described previously (22). A complete list of the BVs used in this study, along with their symbols, is presented in Table 1.

\section{Plaque assay}

The plaque assay was performed as described previously (23) with slight modifications. Briefly, 10-fold serial dilutions of the
BV samples from $10^{5}$ to $10^{7}$ were prepared in the medium. Sf9 cells were stained with 0.4\% trypan blue (Sigma-Aldrich Chemie), counted using the trypan blue exclusion assay using a TC10 automated cell counter (Bio-Rad Laboratories AB, Sundbyberg, Sweden), and diluted to a concentration of $1 \times 10^{6} \mathrm{cell} / \mathrm{s} / \mathrm{mL}$. One milliliter of the cell suspension was added to wells of a BioLite 6 -well plate (ThermoFisher Scientific), and the plate was incubated at $27^{\circ} \mathrm{C}$ for $30-60$ min. The medium was then removed, and-1 $\mathrm{mL}$ aliquots of different dilutions of the BV suspension were added to infect cells. After a 1-h incubation with the BV suspension, the culture medium was removed from each well and replaced with $2 \mathrm{~mL}$ of a $0.7 \%$ agarose (Naxo OÜ, Tartu, Estonia) solution that was maintained at $38.5^{\circ} \mathrm{C}$. The micro-

Table 1. List of baculoviruses (BVs) used for infection and their symbols.

\begin{tabular}{|c|l|l|}
$\begin{array}{c}\text { Virus } \\
\text { symbol }\end{array}$ & \multicolumn{1}{c|}{$\begin{array}{c}\text { Encoded } \\
\text { protein }\end{array}$} & \multicolumn{1}{c|}{$\begin{array}{c}\text { Expression } \\
\text { system type }\end{array}$} \\
\hline$A$ & $\mathrm{D}_{1} \mathrm{R}$ & BEVS \\
\hline $\mathrm{B}$ & $\mathrm{MC}_{4} \mathrm{R}$ & BEVS \\
\hline $\mathrm{C}$ & $\mathrm{Epac}^{\mathrm{H}} \mathrm{H}^{\mathrm{7} 4}$ biosensor & BacMam \\
\hline $\mathrm{D}$ & Epac-S ${ }^{\mathrm{H} 34}$ biosensor & BacMam \\
\hline $\mathrm{E}$ & Epac-S $\mathrm{S}^{\mathrm{H} 88}$ biosensor & BacMam \\
\hline $\begin{array}{l}\text { BEVS: baculovirus expression vector system } \\
\text { for insect cells; BacMam: baculovirus ex- } \\
\text { pression vector system for mammalian cells. }\end{array}$ \\
\hline
\end{tabular}

plate was allowed to cool at room temperature for $20 \mathrm{~min}$. The plate containing the formed gels was sealed and maintained at $27^{\circ} \mathrm{C}$ for $6-10$ days until plaque counting. Plaques were marked, imaged, and counted from the images using ImageJ (https://imagej.nih.gov/ij/).

\section{Samples for CPCSE and ICSE assays} Three-fold serial dilutions from dilution factor $10^{0.30}$ to $10^{5.07}$ of BV were prepared in the medium. Sf9 cells were prepared, counted and diluted to a concentration of $8 \times 10^{5}$ cells $/ \mathrm{mL}$. Two-hundred-fifty microliters of the cell suspension was added to each of the wells of a BioLite 24-well plate (ThermoFisher Scientific) that was maintained at $27^{\circ} \mathrm{C}$ for 20-30 min. Two-hundred-fifty microlitres of the corresponding dilution of the virus suspension was then added to each well. Medium without virus was added to obtain mock-infected cells for comparison. The plate was maintained at $27^{\circ} \mathrm{C}$ until the beginning of the measurements. Plates with other dimensions can be used with according changes to the protocol.

\section{CPCSE assay}

In the CPCSE assay, a Z2 Series Coulter Counter (Beckman-Coulter, Brea, CA) was used to measure the cell diameters as described previously $(11,24)$. This technique 
estimates the volume of small particles by measuring the change in impedance of an electrolyte solution when particles move through a capillary. Assuming the particles are spherical, their diameters can be calculated, and for Sf9 cells, the optimal diameter range was 11-22 $\mu \mathrm{m}$. Twentyfour hours after initiation of the primary infection, Sf9 cells ( $0.5 \mathrm{~mL}$ cell suspension) from the microplate wells were transferred to $9.5 \mathrm{~mL}$ ISOTON II Diluent BeckmanCoulter), resulting in a 20 -fold dilution. The cell diameters were measured, and cells were counted according to the manufacturer's instructions.

\section{ICSE assay}

For the ICSE assay, we used the Cytation 5 imaging microplate reader (BioTek Instruments, Winooski, VT) with the corresponding Gen5 software for parameter control and image acquisition. Imaging was performed in the bright-field mode using a UPLFLN 4x objective lens (WD 17, NA 0.13) (Olympus, Waltham, MA). Four images were taken from each well, each $2000 \mu \mathrm{m}$ away from the well center due to a heterogeneous distribution of cells in the well center. Measurements were performed on all of the wells (4 images/ well) of the plate $24 \mathrm{~h}$ after the initiation of the primary infection of Sf9 cells. For kinetic studies, images were taken using the same imaging parameters at $0.5 \mathrm{~h}$ intervals until $48 \mathrm{~h}$ post-infection. The cell diameters from the obtained images were measured using ICSE-Tools software, which we developed for this purpose. The detailed measurement parameters used for the assay are presented in the Supplementary Material. Documentation for the software, with the code, tutorials, and sample images, can be found at www. gpcr.ut.ee/software.html.

\section{Regression models}

The effective BV concentration was found by fitting the cell diameter dependency to the sample dilution factor. Two threeparameter functions were used to fit the experimental data. GraphPad Prism 5 (GraphPad Software, Inc., La Jolla, CA) was used to fit the data and compare the models using two-way ANOVA with the Bonferroni post-hoc test.

Both functions followed the general form:

$$
D(x)=D_{\text {uninf }}+\left(D_{\text {inf }}-D_{\text {uninf }}\right) * Z(x)
$$

[Eq. 1],

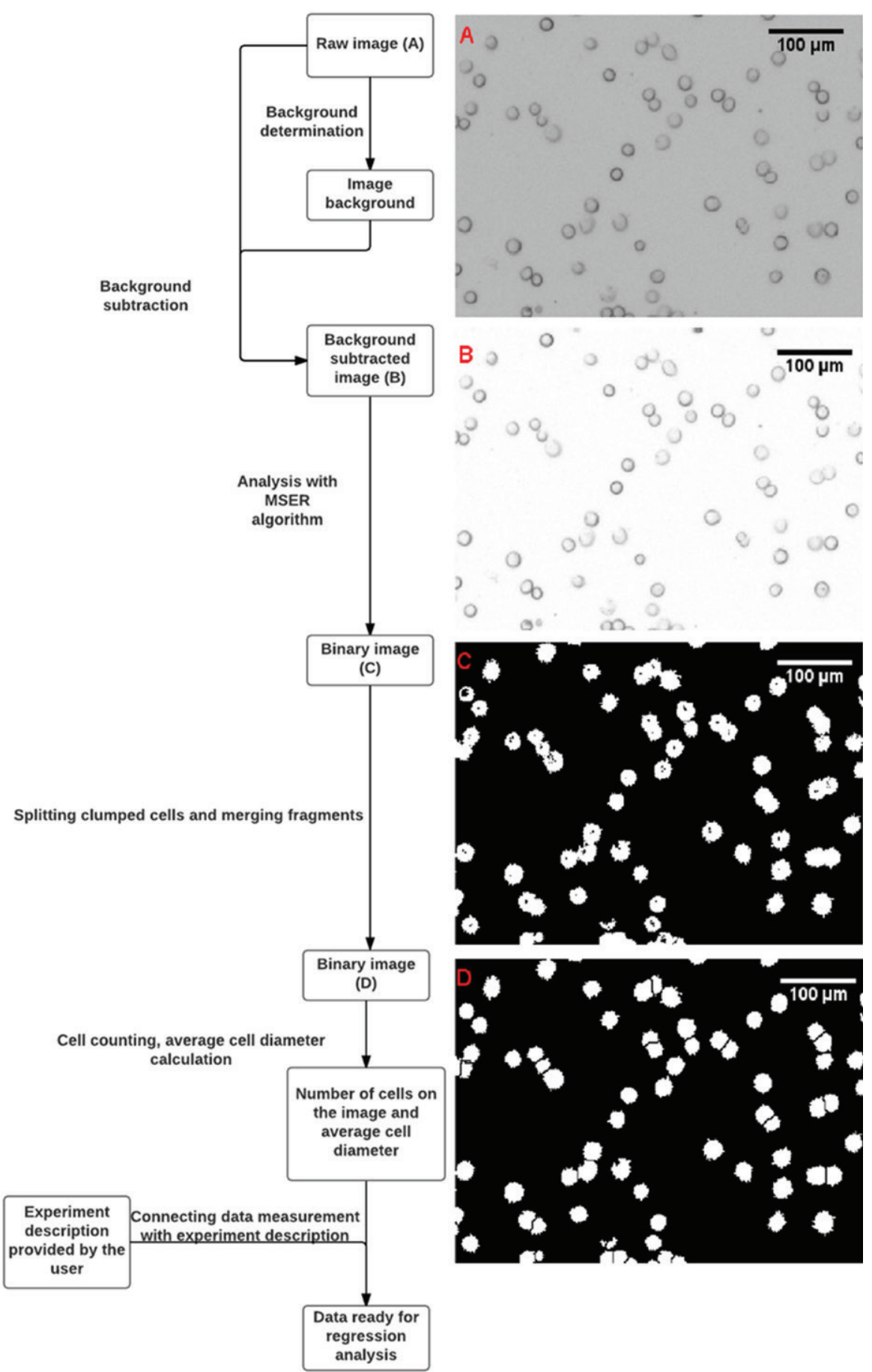

Figure 2. Data flow in the ICSE-Tools software. Image analysis steps: (A) raw image; (B)backgroundsubtracted image; (C) Binary image after applying the maximally stable extremal regions (MSER) algorithm; (D) Binary image after splitting clumped cells and merging fragments. The software counts cells and calculates the average cell diameter, which can be input for further data analysis.

where $D$ is the measured mean cell diameter, $D_{\text {uninf }}$ is the diameter of uninfected cells, $D_{\text {inf }}$ is the diameter of infected cells, $x$ is the dilution factor of the BV sample used, and $Z$ is a function of the dilution factor of the BV sample that predicts the fraction of infected cells. The values of $D_{\text {uninf }}$ and $D_{\text {inf }}$ were found by fitting experimental data. If it is assumed that any cell can be infected by only one BV particle, the $Z(x)$ function can be derived from Reference 18 as:

$$
Z(x)=\frac{1}{1+10^{\log E C_{50}+\log (x)}}
$$

[Eq. 2], 
Table 2. Comparison of virus concentration measured by different methods.

\begin{tabular}{|c|c|c|c|c|c|}
\hline Virus & ICSE assay Model 1 & ICSE assay Model 2 & CPCSE assay Model 1 & CPCSE assay Model 2 & Plaque assay \\
\hline A & $8.21 \pm 0.02$ & $8.18 \pm 0.02$ & $8.19 \pm 0.02$ & $8.41 \pm 0.03^{*}$ & $7.7 \pm 0.2$ \\
\hline B & $8.22 \pm 0.04$ & $8.42 \pm 0.03$ & $8.01 \pm 0.03$ & $8.24 \pm 0.05$ & $7.92 \pm 0.02$ \\
\hline C & $8.30 \pm 0.02^{*}$ & $8.43 \pm 0.03^{* *}$ & $8.1 \pm 0.4$ & $8.2 \pm 0.4^{*}$ & $7.5 \pm 0.2$ \\
\hline D & $8.1 \pm 0.2$ & $8.2 \pm 0.2$ & $7.95 \pm 0.06$ & $8.15 \pm 0.07$ & $7.6 \pm 0.2$ \\
\hline$E$ & $8.4 \pm 0.3^{* *}$ & $8.5 \pm 0.3^{* * *}$ & $7.9 \pm 0.16$ & $8.10 \pm 0.15$ & $7.45 \pm 0.13$ \\
\hline
\end{tabular}

Baculovirus (BV) concentration is expressed as the logarithm of infectious viral particles per milliliter [log (ivp)]. Data from the assays were fitted to Model 1 (Equation $1+$ Equation 2) or to Model 2 (Equation $1+$ Equation 3$)$ and are presented as the mean \pm SEM of three independent experiments carried out in duplicate. Statistically significant differences from the plaque assay: ${ }^{*}(P<0.05),{ }^{* *}(P<0.01),{ }^{* * *}(P$ $<0.001)$. ICSE: image-based cell-size estimation; CPCSE: Coulter principle-based cell-size estimation.

where $E C_{50}$ corresponds to the reciprocal value of the sample dilution factor, where $Z=0.5$ (Model 1).

If it is assumed that several BV particles can infect a single cell, the $Z(x)$ function can be derived from Reference 25 as:

$$
Z(x)=1-\exp \left(-M * 10^{1 / x}\right)
$$

[Eq. 3],

where $M$ is the $\mathrm{MOI}$ of the sample (Model 2).

From Model 1, the virus concentration $c$ expressed as infectious viral particles per milliliter (ivp/mL) can be calculated as:

$$
c=\frac{N}{2 * E C_{50}{ }^{*} V}
$$

[Eq. 4],

where $N$ is the number of cells in a single well at the time of primary infection, and $V$ is the volume of solution in a single well in milliliters.

The concentration of the BV sample is related to the $\mathrm{MOI}$ by the following equation:

[Eq. 5],

$$
c=M * N / V
$$

where $M$ is the $\mathrm{MOI}$ of the sample, $N$ is the number of cells per well, and $V$ is the volume of the solution in a single well in milliliters.

\section{Results and discussion}

\section{Determining cell diameters}

The ICSE assay is based on direct measurement, using a microscope, of cell-size growth upon being infected by BV particles. This approach became more feasible with the introduction of imaging microplate readers, which are capable of automatic bright-field cell imaging using a predetermined measurement protocol. For ICSE, we employed a Cytation 5 microplate reader from BioTek Instruments with the corresponding Gen5 software, but this does not exclude the use of similar systems.

We could not, however, find a suitable software solution to perform the image analyses and data organization steps required by our assay design. To solve this problem, we developed a new MATLAB (The MathWorks, Natick, MA)-based software, called ICSE-Tools, for image analysis and data organization in order to measure the cell diameters. ICSE-Tools is controlled by a straightforward graphical user interface (Figure 1), and documentation, including a user manual and several tutorials, is available at www.gpcr. ut.ee/software.html. ICSE-Tools allows fast and precise detection of cells from bright-field images provided by microscopes or imaging microplate readers. The software recognizes and counts cells and also measures their diameters.

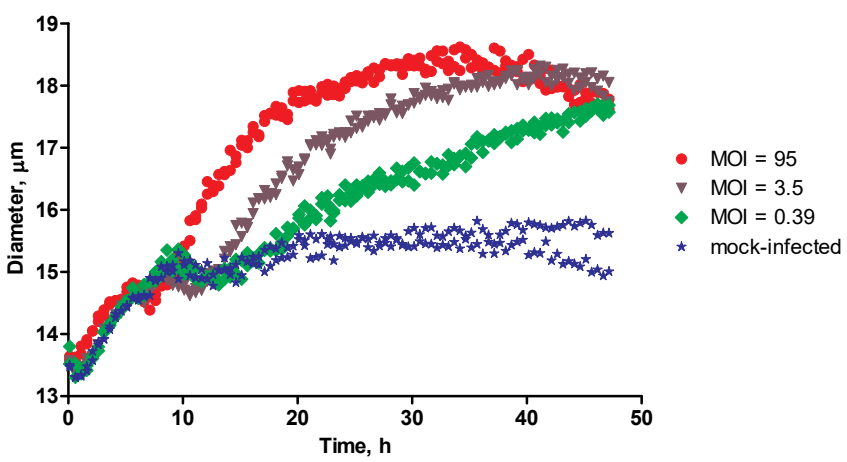

Figure 3. Increase of apparent cell diameter in real time in the presence of virus. Sf9 cells were incubated with different concentrations [expressed as multiplicity of infection (MOI)] of Virus D (Table 1), and the diameters of cells at the indicated time points were measured using the image-based cell-size estimation (ICSE) assay. Data are shown for a representative experiment carried out in duplicate.
The first-ever aliquoting pipet controller

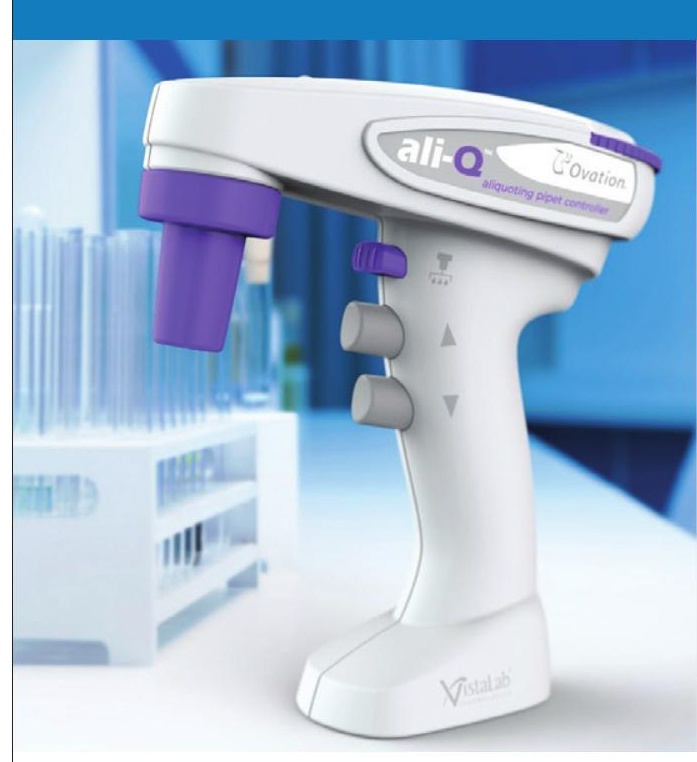

\section{One-button aliquoting}

Get accurate and precise aliquots without the need to "eyeball" the meniscus.

- Faster aliquoting with the press of one button.

- Also aspirates and dispenses like other pipet controllers.

- Uses any size or brand serological pipet.

\section{Use VistaLab ${ }^{\text {TM }}$ Wobble-not $^{\text {TM }}$} serological pipets with ali-Q for the ideal pipetting system.
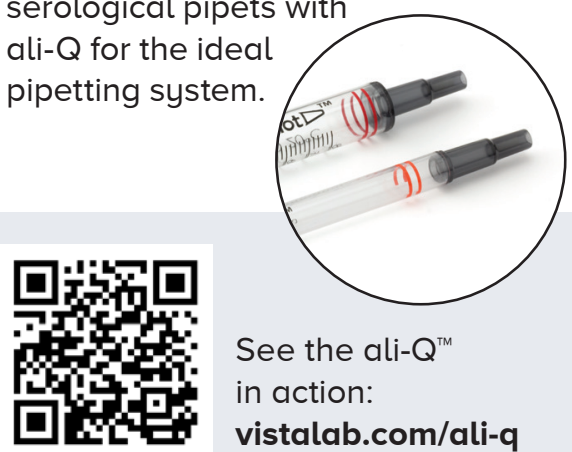

See the ali- $Q^{\mathrm{m}}$ in action: vistalab.com/ali-q

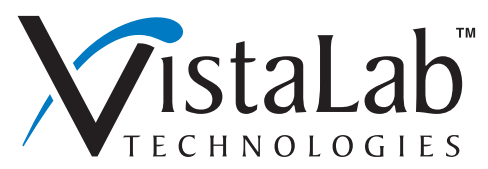
Think Differently. Feel Better. 
The principal steps for ICSE-Tools image analysis and data organization are presented as a flowchart in Figure 2. Analysis starts from Open Microscopy Environment Tagged Image File Format images captured by the imaging device (Figure 2A). The first image analysis step involves a background calculation and subtracting this measurement from the original image, thus obtaining a background-corrected image (Figure 2B). It is important to note that this step is especially critical if data from different experiments, assay systems, or equipment are to be compared. In the next step, primary cell recognition is performed. A maximally stable extremal regions (MSER) algorithm is used to differentiate background pixels from the foreground, in this case, a cell, resulting in a binary image (Figure 2C). In the third step, errors associated with cell aggregation and uneven illumination are corrected. Here, aggregated cells are split, over-segmented cells are merged based on the expected shapes as well as the minimal and maximal sizes of cells, and holes are filled. This step, which was adapted from Reference 26, greatly increases the accuracy of the cell diameter analysis. Next, cells from the image are counted (Figure 2D), and cell diameters are calculated. Cell diameters from all of the images are then organized based on the experimental design and can be exported as user-defined tables to other software for further analysis (e.g., regression, statistics). The image analysis was performed using a conventional desktop computer with four Intel Core i7-4820K CPU 3.70 GHz processors, indicating that ICSE-Tools does not require specialized computer hardware.

Using bright-field images instead of fluorescence images for cell detection has several advantages, as there is no need for additional staining agents, fluorescent dyes or the expression of fluorescent proteins; this means samples are not influenced by additional chemicals or the expression of foreign proteins. Of course, bright-field image analysis also has some weaknesses: the low contrast between cells and significant background levels makes it harder to distinguish cells from the background. However, in recent years several algorithms, such as MSER and an active contours-based approach $(26,27)$, have solved these problems;

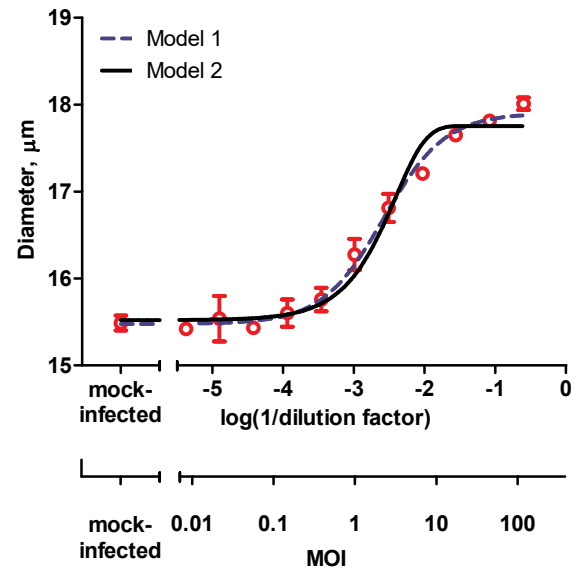

Figure 4. Dependence of cell diameter on virus concentration. Sfg cells were incubated with different concentrations [multiplicity of infection (MOl) values] of Virus $D$ (Table 1). The diameters of the cells were measured after a $24 \mathrm{~h}$ incubation using the image-based cell-size estimation (ICSE) assay and are presented as the mean \pm SD of a representative experiment carried out in duplicate. The concentrations of the virus are presented as $\log (1 /$ dilution factor) and $\mathrm{MOI}$. The data were fitted to Model 1 (Equation 1 + Equation 2; blue dashed line) and to Model 2 (Equation 1 + Equation 3; black solid line).

therefore this approach was suitable for the ICSE assay.

\section{Monitoring cell diameters}

during infection

Imaging does not significantly affect the health of cells, since the measuring chamber is thermostated, and images are taken under low-light conditions. Therefore, cellular processes can be monitored in real time. Because of the speed of the microscope, images can be taken at sufficiently small intervals of time, and automated image analysis ensures that the results can be obtained reasonably quickly. It is known that cell diameter changes are characteristic of BV infection (28). This allows for quantification of the effective BV concentration used to infect cells. We monitored the change in diameter of Sf9 cells in the presence of different amounts of BVs, finding that the growth of cells depended on the time of measurement and the BV concentration (Figure 3). Cell diameter increases were faster in the presence of BVs and plateaued at a higher level compared to uninfected cells. It is important to note that the diameter of non-infected cells (mock-infected) also increased with time, but this may not be connected with the increase of the cell volume and instead may be due to cell flattening during the experiment. Therefore, to rule out the effects of flattening, measurements must be performed against mock-infected control cells.

\section{Determining BV concentration}

The dependence of the cell diameters on the virus concentration allows for the quantification of active BVs. Since determining infection kinetics takes unreasonable amounts of resources (equipment time, data analysis) for routine analysis, a static endpoint approach was proposed for further study, and the optimal measurement time point was found to be $24 \mathrm{~h}$ after primary infection for all of the BVs studied (data not shown). There are several potential models for how BVs affect the properties of cells, but for this study, we used two variants of Equation 1, which describe the dependence between the BV sample dilution factor and cell diameter. The first model (Model 1: Equation 1 + Equation 2) states that healthy uninfected cells have a specific average diameter, whereas cells infected with a BV particle grow to a larger average cell diameter, and it is assumed that each cell can be infected by only a single BV particle (18). The second model (Model 2: Equation $1+$ Equation 3) uses a similar approach, but takes into account the possibility that one cell can be infected by several BV particles (25). An example of the dependence of the diameters of Sf9 cells on the dilution factor of the BVs is shown in Figure 4. Dependence is clearly sigmoidal, and both equations revealed similar fits when the virus concentration was low. These results are expected, since the probability that one cell is infected by several viral particles in this case is low.

A larger difference between fits can be seen at an approximately 100 -fold BV dilution, in which case the difference in the MOls becomes statistically significant; therefore, the models predict somewhat different cell diameters at this dilution. This is important for the general characterization of the infection processes, while the MOls calculated from these curves did not differ significantly from one another $(P>0.05)$ in the case of all of the BVs studied. Thus, due to the robustness of the ICSE method, 
both equations gave similar results and are suitable for determination of the BV concentration. Of course, for more insight into the mechanism of infection, more complex models must be used (29).

Assay validation and comparison of methods

Data presented for Epac-S $\mathrm{S}^{\mathrm{H} 134}$ biosensor BacMam virus (Virus D in Table 1) indicate that the ICSE method allows for precise determination of BV concentration. However, the question of how universal the assay is and how compatible it is with other methods remains.

To further validate the ICSE assay, we used five different BVs, two for use in BEVS and three for use in the BacMam system (Table 1), and determined their concentrations using three different assays: the plaque assay, the CPCSE assay, and the ICSE assay. For the CPCSE and ICSE assays, the two previously described regression models, Models 1 and 2, were used to estimate the BV concentration. The results are presented in Table 2. There were no significant differences between the cell diameter-based assays for any of the BV samples studied. The choice of model also did not significantly change the results of the ICSE and CPCSE assays. However, the plaque assay revealed a relatively lower concentration for all BVs studied. This difference between diameter-based methods and plaque assays has been described in earlier studies (16) and is connected with the different nature of these assays. From a practical point of view, the ICSE assay, unlike CPCSE assay, can be automated and allows for measurement of a sample at several time points without interruption of cellular processes.

In conclusion, ICSE-Tools can be used for cell diameter-based BV quantification as well as for other studies in which the morphological parameters of cells have to be monitored. These morphological changes in cells or statistical changes in cell populations are indicative of several biological and toxicological phenomena (30-32). ICSE-Tools can currently measure the cell diameter distribution, area, circularity, and confluence, but the software could also be modified to calculate other parameters as well. ICSE-Tools is written in MATLAB and is distributed as free software under the GNU GPL. It is available at www.gpcr.ut.ee/software. html.

\section{Authors contributions}

All authors of this report have directly participated in the planning of the study, analysis of the data, and preparation of the manuscript. The experiments were performed and software was developed by T.L. The manuscript was written by T.L. and A.R. All authors have read the final version of the manuscript and approved its submission.

\section{Acknowledgments}

We thank Anni Allikalt and Maris-Johanna Tahk for technical assistance, preparing the baculovirus samples and testing ICSE-Tools; Reet Link, Olga Mazina, Santa Veikšina for preparing the baculovirus samples; and Prof. Arnold Kristjuhan for support in using the CPCSE assay. We also thank all of the people whose open source code was used in ICSE-Tools. The work was financed by the Estonian Ministry of Education and Science (IUT20-17) and by the European Union through the European Regional Development Fund (Competence Centre on Health Technology, EU48695).

\section{Competing interests}

The authors declare no competing interests.

\section{References}

1. Baldi, L., D.L. Hacker, M. Adam, and F.M. Wurm. 2007. Recombinant protein production by large-scale transient gene expression in mammalian cells: state of the art and future perspectives. Biotechnol. Lett. 29:677-684.

2. Cervera, L., S. Gutierrez-Granados, N.S. Berrow, M.M. Segura, and F. Godia. 2015. Extended gene expression by medium exchange and repeated transient transfection for recombinant protein production enhancement. Biotechnol. Bioeng. 112:934946.

3. Kost, T.A. and C.W. Kemp. 2016. Fundamentals of baculovirus expression and applications. Adv Exp Med Biol. 896:187-97.

4. Lindley, K.M., J.L. Su, P.K. Hodges, G.B. Wisely, R.K. Bledsoe, J.P. Condreay, D.A. Winegar, J.T. Hutchins, and T.A. Kost. 2000. Production of monoclonal antibodies using recombinant baculovirus displaying gp64-fusion proteins. J. Immunol. Methods 234:123-135

5. Paul, A., A. Hasan, L. Rodes, M. Sangaralingam, and S. Prakash. 2014. Bioengineered baculoviruses as new class of therapeutics

\section{The first-ever}

ergonomic

serological pipet

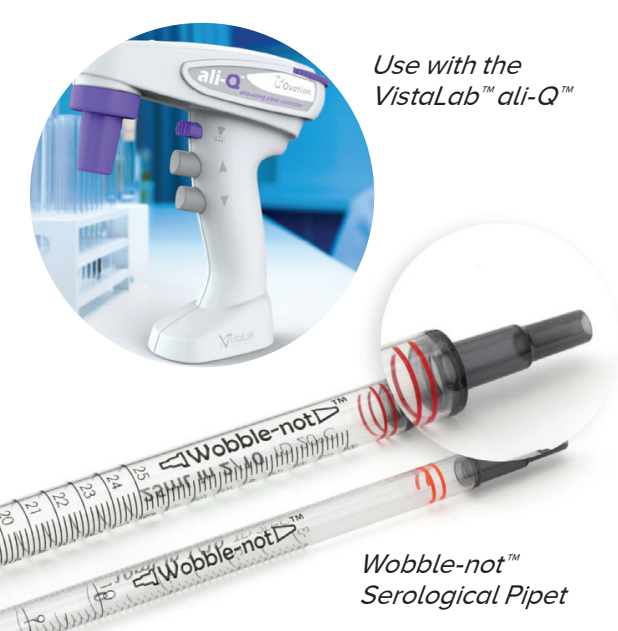

\section{Lose the Wobble, Lose the Drip!}

Other serological pipets require jamming to get the pipet into your controller or risk wobble and dripping.

- Increase your accuracy and precision with Wobble-not ${ }^{\mathrm{T}}$.

- Less fatigue due to the Wobble-not ${ }^{\text {tw }}$ ergonomic design.

- Use with any pipet controller.

For the ideal pipetting system, use with VistaLab ali-Q aliquoting pipet controller.

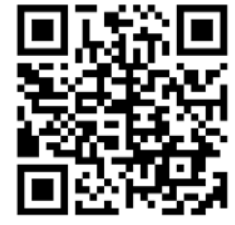

Get your free sample pack vistalab.com/ wobble-not

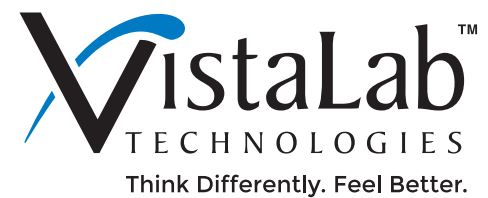

vistalab.com/wobble-not • +1 914-244-6226

2 Geneva Road, Brewster NY 10509 
